40th "Jaszowiec" International School and Conference on the Physics of Semiconductors, Krynica-Zdrój 2011

\title{
Quantum Monte Carlo vs. Density Functional Methods for the Prediction of Relative Energies of Small $\mathrm{Si}-\mathrm{C}$ Clusters
}

\author{
N. Gonzalez Szwacki And J.A. Majewski
}

Institute of Theoretical Physics, Faculty of Physics, University of Warsaw, Hoża 69, 00-681 Warsaw, Poland

\begin{abstract}
In the present paper, we assess the accuracy of popular and widely used approaches based on density functional theory by relating them to the most accurate at present quantum Monte Carlo calculations. As the test case, we consider the relative stability of small $\mathrm{Si}_{n} \mathrm{C}_{m}$ isomers. We find out that none of the studied DFT approaches employing local, semilocal, or even hybrid functionals are able to predict correctly the relative stability of the isomers.
\end{abstract}

PACS: 31.15.E-, 36.40.-c

\section{Introduction}

$\mathrm{Si}-\mathrm{C}$ clusters are involved in many technological processes such as chemical vapor deposition used for the manufacture of $\mathrm{SiC}$ thin films. Knowledge of their properties is therefore important for the development of new materials for electronics-related applications. Numerous experimental and theoretical studies have been carried out to investigate equilibrium geometries, relative energies, and vibrational frequencies of small $\mathrm{Si}_{n} \mathrm{C}_{m}$ clusters [1-3]. On a larger scale the computer simulation of $\mathrm{SiC}$ crystal growth depends on the quality of the effective potential that is used to model the interactions between the involved atoms. To obtain such a potential model for a given system, one usually fits the parameters of some functional form of the potential to the first-principles calculations or to experimental data. The question arises how reliable are the widely used density functional theory (DFT) based approaches for the determination of these semi-empirical potentials, and how accurately they are able to predict, for example, the relative energies of small cluster isomers. In a recent study, Hsing and coworkers [4] have shown that the DFT methods are able to describe correctly the relative stability of monoatomic metallic aluminum and copper cluster isomers, but fail in predicting the relative stability of covalently bonded carbon and boron isomers. For benchmark calculations, the diffusion Monte Carlo (DMC) method was used, which is the most accurate method for system of interacting electrons known up to now.

The aim of this work is to examine the accuracy of various DFT exchange-correlation (XC) functionals in predicting the relative energies of small $\mathrm{Si}_{n} \mathrm{C}_{m}$ cluster isomers and to provide new benchmark data by performing very accurate DMC calculations for these clusters. We compare our DMC results with DFT ones obtained using the local density approximation (LDA), generalized gradient approximation (GGA), and a hybrid functional that combines Hartree-Fock (HF) exchange with DFT exchange-correlation.

\section{Calculation details}

The initial cluster geometries are taken from Fig. 3 in Ref. [1]. However, we have also performed our own search for the most stable candidates at the DFT level.

For the DFT calculations, we use the GAMESS-US [5] package. The LDA, GGA, and $\mathrm{HF} / \mathrm{DFT} \mathrm{XC}$ functionals that are tested are: the Slater exchange and Vosko-WilkNusair local correlation (SVWN), the Perdew-BurkeErnzerhof semilocal exchange-correlation (PBE), and the Becke three parameter hybrid functional (B3LYP), respectively. All the clusters are fully relaxed using the 6-311++G(d,p) basis set.

The QMC calculations are done using the QWalk package [6] in two steps. The first step consists of optimizing the trial many-body wave function by performing the variational Monte Carlo (VMC) calculations. The trial wave function is of the Slater-Jastrow form. The Slater determinants are constructed using B3LYP orbitals generated using the GAMESS-US code with previously optimized geometries within the B3LYP $/ 6-311++\mathrm{G}(\mathrm{d}, \mathrm{p})$ level of theory. For the QMC calculations, we use Gaussian basis sets with effective core potentials [7]. In the second step, we do fixed-node DMC calculations with previously optimized trial wave functions. We use a time step of 0.005 a.u.

\section{Results and discussion}

In Fig. 1 we compare the total energies obtained using the DMC with those obtained using the DFT approaches for three sets of four cluster isomers. The energies, $E_{(i)}$, plotted in the figure for each of the four theoretical methods employed are given relative to the average energy of four isomers of a $\mathrm{Si}_{n} \mathrm{C}_{m}$ cluster by the following formula:

$$
E_{(j)}=E_{(j)}^{\mathrm{tot}}-\sum_{i=1}^{4} E_{(i)}^{\mathrm{tot}} / 4,
$$

where $E_{(j)}^{\text {tot }}$ is the total energy of isomer $(j)$ obtained using 


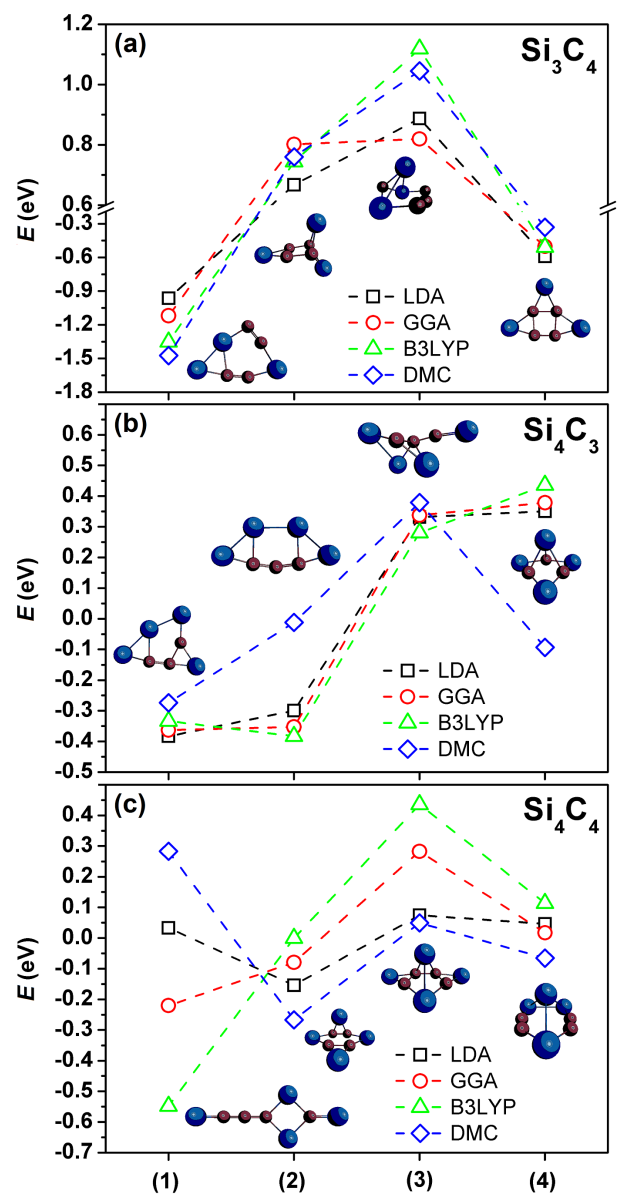

Fig. 1. Energies (defined by Eq. (1)) for the isomers of (a) $\mathrm{Si}_{3} \mathrm{C}_{4}$, (b) $\mathrm{Si}_{4} \mathrm{C}_{3}$, and (c) $\mathrm{Si}_{4} \mathrm{C}_{4}$ clusters. DMC error bars are about $0.1 \mathrm{eV}$. The structure of the isomers is shown. Dark large blue and small brown balls represent silicon and carbon atoms, respectively. Let us note that in part (a) the isomers of the $\mathrm{Si}_{3} \mathrm{C}_{4}$ cluster labeled as (1) and (4) are planar, whereas in part (b) isomers (1) and (2) of the $\mathrm{Si}_{4} \mathrm{C}_{3}$ cluster are planar, and in part (c) only isomer (1) of the cluster $\mathrm{Si}_{4} \mathrm{C}_{4}$ is planar.

one of the theoretical approaches. Taking the DMC results as a reference, we can see in Fig. 1a that in the case of the $\mathrm{Si}_{3} \mathrm{C}_{4}$ cluster, the LDA and GGA approximations underestimate the relative energy, $\Delta E=E_{(4)}-E_{(1)}$, of the planar isomers labeled as (1) and (4). Moreover, the GGA approximation is unable to capture correctly the relative energy of the $3 \mathrm{D}$ isomers indicated as (2) and (3). The B3LYP hybrid functional gives the closest results to those computed with DMC. In Fig. 1b presenting results for $\mathrm{Si}_{4} \mathrm{C}_{3}$ cluster, one can see that from all the DFT approaches only LDA is capable to predict the correct energy order $E_{(1)}<E_{(2)}$ for isomers (1) and (2) of $\mathrm{Si}_{4} \mathrm{C}_{3}$, but there is still a large difference in comparison to the DMC results. The most notable demonstration of the problem of the DFT approximate schemes in describing the proper energy order of clusters is shown for $\mathrm{Si}_{4} \mathrm{C}_{4}$ cluster in Fig. 1c. The popular B3LYP functional clearly predicts that the planar isomer of this cluster is the most energetically favorable among the four studied, whereas the true minimum structure is isomer (2) according to the LDA and DMC results.

To quantify to which extent the DFT approaches can predict the relative stability of the cluster isomers, we perform the correlation analysis introduced in Ref. [4]. The correlation (corr) between the DFT and DMC energies and the relative amplitude $(L)$ is given by

$$
\left\{\begin{array}{l}
\text { corr }=\frac{\boldsymbol{D}^{\mathrm{DFT}} \cdot \boldsymbol{D}^{\mathrm{DMC}}}{\left(\left|\boldsymbol{D}^{\mathrm{DFT}}\right|\left|\boldsymbol{D}^{\mathrm{DMC}}\right|\right)}, \\
L=\frac{\left|\boldsymbol{D}^{\mathrm{DFT}}\right|}{\left|\boldsymbol{D}^{\mathrm{DMC}}\right|},
\end{array}\right.
$$

where $\boldsymbol{D}=\left(E_{(1)}, E_{(2)}, E_{(3)}, E_{(4)}\right)$ is a vector of four elements defined by Eq. (1). The DFT and DMC energies $\left(E_{(j)}\right)$ are identical only if corr $=1$ and $L=1$.

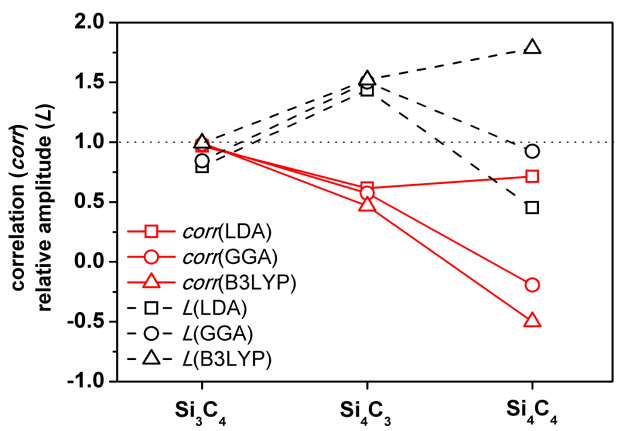

Fig. 2. Correlation (corr) between DFT and DMC energies and the relative amplitude $(L)$ defined by Eq. (2), plotted for the three families of clusters.

For our clusters and DFT approaches, the computed values of corr and $L$ are plotted in Fig. 2. From this figure (and also from Fig. 1), we can see that for the $\mathrm{Si}_{3} \mathrm{C}_{4}$ cluster the B3LYP energy values are very close to those obtained with DMC since both corr and $L$ are close to one. For the same clusters LDA and GGA correlate well with DMC, however those approaches tend to predict smaller energy differences than DMC since for both $L<1$. From Fig. 2, we can also see that for the $\mathrm{Si}_{4} \mathrm{C}_{3}$ clusters the DFT methods give similar results, namely corr $<1$ and $L>1$. Finally, for the $\mathrm{Si}_{4} \mathrm{C}_{4}$ clusters only LDA correlates reasonably well with DMC, however underestimates energy differences $(L<1)$.

\section{Conclusions}

Our studies strongly suggest that the calculations of covalently bonded $\mathrm{Si}_{n} \mathrm{C}_{m}$ clusters performed within the local LDA, the semilocal GGA or even the hybrid functional should be used with caution. The LDA functional gives rather similar trends in relative stability of the isomers as the DMC approach does, which may suggest that it is qualitatively more accurate for the prediction of the properties of small $\mathrm{Si}_{n} \mathrm{C}_{m}$ clusters than the GGA or the hybrid functional. 
From the present work and also from previous investigations [1-3], an important remark can be drawn. When predicting the structures of larger $\mathrm{Si}_{n} \mathrm{C}_{m}$ clusters, the knowledge of the structure of clusters of a smaller size is of limited help. Even the structures of the clusters considered in this work cannot be obtained by simply adding (removing) an atom to (from) an isomer of a smaller (larger) size. This lack of simple building blocks makes the prediction of the structures of $\mathrm{Si}_{n} \mathrm{C}_{m}$ clusters particularly hard.

\section{Acknowledgments}

The studies were carried out within the SiCMAT project financed under the European Funds for Regional Development (contract No. UDA-POIG.01.03.01$-14-155 / 09)$.

\section{References}

[1] M. Bertolus, F. Finocchi, P. Millie, J. Chem. Phys. 120, 10853 (2004).

[2] J. Hou, B. Song, J. Chem. Phys. 128, 154304 (2008).

[3] Y.-Z. Lan, Y.-L. Feng, J. Chem. Phys. 131, 054509 (2009).

[4] C.R. Hsing, C.M. Wei, N.D. Drummond, R.J. Needs, Phys. Rev. B 79, (2009).

[5] M.W. Schmidt, M. Duncalf, A. Gaenko, M. Gordon, GAMESS-US, Version: 1 OCT 2010 (R1) http://www.msg. chem.iastate.edu/gamess/ .

[6] L.K. Wagner, M. Bajdich, L. Mitas, QWalk, Version: 0.95.0, http://www.qwalk.org/ .

[7] For silicon we use "LANL08d" from the basis set repository https://bse.pnl.gov/; for carbon we use a basis set from the examples supplied with the package. 